\title{
Microscopic Phase-Field Simulation for the Influence of Interatomic Potential on the Precipitation Process of $\mathrm{Ni}_{75} \mathrm{Al}_{14} \mathrm{Mo}_{11}$ Alloy
}

\author{
Qing-Shuang $\mathrm{Ma}^{1} \cdot$ Yu-Chun $\mathrm{Jin}^{1} \cdot$ Yu-Hong Zhao ${ }^{1} \cdot$ Huai-Ming Sui ${ }^{2} \cdot$ Pu Zhang $^{1}$
}

Received: 11 January 2016/Revised: 9 May 2016/Published online: 22 August 2016

(C) The Chinese Society for Metals and Springer-Verlag Berlin Heidelberg 2016

\begin{abstract}
The process of $\gamma^{\prime}$ phase precipitating from $\mathrm{Ni}_{75} \mathrm{Al}_{14} \mathrm{Mo}_{11}$ is studied by a computational simulation technique based on microscopic phase-field kinetics model. We studied the phase transformation with the purpose of clarifying the influence of the nearest interatomic potential $V_{\mathrm{Ni}-\mathrm{Al}}$ (the nearest interatomic potential) on the precipitation process of $\gamma^{\prime}$ phase. The result demonstrates that there are two kinds of ordered phases, respective $\mathrm{L} 1_{0}$ and $\mathrm{L}_{2}$ in the early stage, and $\mathrm{L}_{0}$ phase transforms into $\mathrm{L}_{2}$ phase subsequently. For $\mathrm{L}_{2}$ phase, $\mathrm{Ni}$ atoms mainly occupy $\alpha$ site (face center positions), while $\mathrm{Al}$ and Mo atoms occupy $\beta$ sites (the vertex positions). When $V_{\mathrm{Ni}-\mathrm{Al}}$ is increased by $10 \mathrm{MeV}$, the occupation probability of $\mathrm{Ni}$ atoms on $\alpha$ sites and $\mathrm{Al}$ atoms on $\beta$ sites are enhanced. Enhanced $V_{\mathrm{Ni}-\mathrm{Al}}$ facilitates clustering and ordering of $\mathrm{Al}$ atom, which promotes the formation of the $\gamma^{\prime}$ phase. At last, the simulation result was discussed by employing the thermodynamic stability.
\end{abstract}

KEY WORDS: Interatomic potential; Phase transformation; Microscopic phase-field; Occupation probability

\section{Introduction}

Interatomic potential and molecular dynamics are of great significance to the development of materials science and technology. The atomic simulation of the materials is based on the interatomic potential, and by this method we can study the properties of the materials at the atomic level and the change process when external conditions changed.

Interatomic potential decides interatomic ordering energy. While the interatomic ordering energy is related to the parameters such as electron density, temperature,

Available online at http://link.springer.com/journal/40195.

Yu-Hong Zhao

zyh388@sina.com

1 School of Materials Science and Engineering, North University of China, Taiyuan 030051, China

2 Yangzhou Feng Ming Metal Products Co., Ltd, Yangzhou 225117, China electric field intensity and its variation trend along with plus potential field is more complicated. Changes of interatomic potential can be used to study the impact of external fields (electric field, magnetic field, etc.) on the precipitation of the alloy indirectly. Ustinovshikov [1] studied the microstructure evolution of the $\mathrm{Ni}_{75} \mathrm{Mo}_{20} \mathrm{Al}_{15}$ alloy at the temperatures ranging from 600 to $1600{ }^{\circ} \mathrm{C}$ using transmission electron microscopy. By the phase-field method, Miao et al. [2] investigated the relationship between occupation probability changes of $\mathrm{Al}$ and $\mathrm{Cr}$ atoms on (100) and (200) planes, respectively, and the structure evolution of $\mathrm{Ni}_{3}(\mathrm{AlCr})$ and found that there are twice in situ transformation during the very early stage. $\mathrm{Lu}$ et al. [3] investigated the pre-precipitation process of $\mathrm{Ni}-$ Al-Cr alloy using the microscopic phase-field kinetics model and found that temperature plays a significant effect on the pre-precipitated phase with $\mathrm{L1}_{0}(M=1)$ structure. With increasing temperature, the incubation period and existent time of $\mathrm{L1}_{0}(M=1)$ pre-precipitated phase are prolonged. It is also found that elastic strain energy has a little effect on the precipitation sequence of $\mathrm{Ni}-6.8$ at.\% 
Al-18.2 at.\% $\mathrm{Cr}$ alloy [4]. With elastic strain energy increasing, the occupation probabilities of $\mathrm{Cr}$ atoms at $\beta$ sublattices and $\alpha$ sublattices in $\mathrm{DO}_{22}$ phase both increase, the occupation probabilities of $\mathrm{Cr}$ atoms at $\beta$ sublattices in $\mathrm{L}_{2}$ phase decrease and that of $\alpha$ sublattices in $\mathrm{L}_{2}$ phase increase. By graphical demonstration of the anti-site defect-diffusion mechanism, Zhang et al. [5] explained the contribution and the potential harm of anti-site defect to the intermetallic structure materials. Dong et al. [6, 7] studied the formation and transformation of the pre-precipitated phase in the early stage of $\mathrm{Ni}_{0.75} \mathrm{Al}_{0.05} \mathrm{Fe}_{0.2}$ alloy by using the interatomic potential during early-stage precipitation. Zhao et al. [8] studied $\mathrm{Cr}$ substitution behavior in $\mathrm{Ni}_{75}$ $\mathrm{A} 1_{15} \mathrm{Cr}_{10}$ alloy aged at $1073 \mathrm{~K}$ using the microscopic phase-field model and found that different interatomic potential can change the $\mathrm{Cr}$ occupation probability in $\alpha$ and $\beta$ sites of $\gamma^{\prime}$ phase.

Pasianot et al. [9] developed an interatomic potential method to investigate the microstructure evolution and point defects of the $\mathrm{Fe}-\mathrm{Cu}$ system based on the embedded atom method (EAM). Oramus et al. [10] simulated the precipitation mechanism of $\mathrm{Ni}_{3} \mathrm{Al}$ alloy using MD method on the basis of embedded atom interaction potential.

Although previous studies reported the influence of interatomic potential on the precipitation process in different alloys, few studies have been done about $\mathrm{Ni}_{75} \mathrm{Al}_{14} \mathrm{Mo}_{11}$ alloy, and almost no reasonable explanation is there on how interatomic potential influence phase precipitation.

In this paper, considering the effect of external field on the atomic ordering behavior, we investigated how Ni-Al interatomic potential influences the precipitation process of the $\gamma^{\prime}$ phase in $\mathrm{Ni}_{75} \mathrm{~A}_{14} \mathrm{Mo}_{11}$ alloy aged at $1073 \mathrm{~K}$, including ordering and clustering of $\mathrm{Al}$ and $\mathrm{Mo}$ as well as the incubation period of $\gamma^{\prime}$ phase and occupation probability of different atoms in $\alpha$ and $\beta$ sites. By studying the influence of interatomic potential, we hope to design the microstructure of the alloy by control external field.

\section{Phase-Field Model}

The microscopic phase-field kinetics model based on Ginzburg-Landau theory was found by Khachaturyan [11] and developed by Chen [12-15]. It is a deterministic phasefield method which can describe all diffusion processes, including atomic clustering, ordering, boundary migration, growing and coarsening by using occupation probability $P(r, t)$, indicating occupation probability on lattice $\boldsymbol{r}$ at time $t$, and it is a discrete lattice form of Cahn-Hilliard diffusion equation [16]. In addition, this model can produce microstructures, composition and order degree changes of system in early precipitation stage thoroughly. The kinetic equation of ternary system is written as

$$
\left\{\begin{array}{l}
\frac{\mathrm{d} P_{A}(r, t)}{\mathrm{d} t}=\frac{1}{k_{\mathrm{B}} T} \sum_{r^{\prime}} \\
\quad \times\left[L_{A A}\left(r-r^{\prime}\right) \frac{\partial F}{\partial P_{A}\left(r^{\prime}, t\right)}+L_{A B}\left(r-r^{\prime}\right) \frac{\partial F}{\partial P_{B}\left(r^{\prime}, t\right)}\right] \\
\frac{\mathrm{d} P_{B}(r, t)}{\mathrm{d} t}=\frac{1}{k_{\mathrm{B}} T} \sum_{r^{\prime}} \\
\quad \times\left[L_{B A}\left(r-r^{\prime}\right) \frac{\partial F}{\partial P_{A}\left(r^{\prime}, t\right)}+L_{B B}\left(r-r^{\prime}\right) \frac{\partial F}{\partial P_{B}\left(r^{\prime}, t\right)}\right]
\end{array} .\right.
$$

Equation (1) is a microscopic Langevin equation, where $L_{\alpha \beta}\left(r-r^{\prime}\right)$ is the atom transition probability constant of $\alpha$ and $\beta$, which changes from lattice $r$ to $r^{\prime}$; and $\alpha, \beta=A, B$ or $C ; k_{B}$ is Boltzmann constant; $t$ is time step; $T$ is the temperature; $P_{A}(r, t), P_{B}(r, t)$ and $P_{C}(r, t)$ stand for occupation probabilities of $A, B$ and $C$ at a certain time step, and $P_{C}(r, t)=1-P_{A}(r, t)-P_{B}(r, t) ; F$ is the total free energy of system, it comes from the mean field theory

$$
\begin{aligned}
F= & -\frac{1}{2} \sum_{r} \sum_{r^{\prime}}\left[V_{A B}\left(r-r^{\prime}\right) P_{A}(r) P_{B}\left(r^{\prime}\right)\right. \\
& \left.+V_{B C}\left(r-r^{\prime}\right) P_{B}(r) P_{C}\left(r^{\prime}\right)+V_{A C}\left(r-r^{\prime}\right) P_{A}(r) P_{C}\left(r^{\prime}\right)\right] \\
& +k_{\mathrm{B}} T \sum_{r}\left[P_{A}(r) \ln \left(P_{A}(r)\right)+P_{B}(r) \ln \left(P_{B}(r)\right)\right. \\
& \left.+P_{C}(r) \ln \left(P_{C}(r)\right)\right],
\end{aligned}
$$

$V_{A B}\left(r-r^{\prime}\right)=W_{A A}\left(r-r^{\prime}\right)+W_{B B}(r-r)-2 W_{A B}\left(r-r^{\prime}\right)$

$V_{B C}\left(r-r^{\prime}\right)=W_{B B}\left(r-r^{\prime}\right)+W_{C C}\left(r-r^{\prime}\right)-2 W_{B C}\left(r-r^{\prime}\right)$

$V_{A C}\left(r-r^{\prime}\right)=W_{A A}\left(r-r^{\prime}\right)+W_{C C}\left(r-r^{\prime}\right)-2 W_{A C}\left(r-r^{\prime}\right)$,

where $V_{\alpha \beta}$ is a useful interaction energy among atoms and in the Fourier space is $V(k) ; W_{\alpha \beta}\left(r-r^{\prime}\right)$ is the elastic interaction energy and in Fourier space is $B(e)$. In this model, the fourth nearest-neighbor interatomic potential is adopted. $V_{\alpha \beta}^{1}, V_{\alpha \beta}^{2}, V_{\alpha \beta}^{3}$, and $V_{\alpha \beta}^{4}$ are the first-, second-, third- and fourth-nearest interatomic potentials between $\alpha$ and $\beta$, respectively. Then, substituting them into the FCC, the reciprocal space is as follows:

$$
\begin{aligned}
V_{\alpha \beta}(\vec{k})= & 4 V_{\alpha \beta}^{1}(\cos \pi h \cdot \cos \pi k+\cos \pi h \cdot \cos \pi l+\cos \pi k \cdot \cos \pi l) \\
& +2 V_{\alpha \beta}^{2}(\cos 2 \pi h+\cos 2 \pi k+\cos 2 \pi l) \\
& +8 V_{\alpha \beta}^{3}(\cos 2 \pi h \cdot \cos \pi k \cdot \cos \pi l+\cos \pi h \cdot \cos 2 \pi k \cdot \cos \pi l) \\
& +\cos \pi h \cdot \cos \pi k \cdot \cos 2 \pi l)+4 V_{\alpha \beta}^{4}(\cos 2 \pi h \cdot \cos 2 \pi k \\
& +\cos 2 \pi h \cdot \cos 2 \pi l+\cos 2 \pi k \cdot \cos 2 \pi l)
\end{aligned}
$$

where $h, k$, and $l$ are reciprocal lattice sites obtained from the following:

$$
\vec{k}=\left(k_{x}, k_{y}, k_{z}\right)=h{\overrightarrow{a_{1}}}^{*}+k{\overrightarrow{a_{2}}}^{*}+l{\overrightarrow{a_{3}}}^{*}
$$


where $\vec{a}_{2}^{*}, \vec{a}_{2}^{*}$, and $\vec{a}_{3}^{*}$ are the unit reciprocal lattice vectors of the FCC lattice structure along three directions.

A Fourier transform was applied to Eqs. (1) and (2), and the free energy was added to the microscopic Langevin equation in the Fourier space. We solved the equation using the Euler method and obtained the occupation probabilities of the atoms at different time steps, and the evolution morphology of the precipitates is obtained according to the variation of occupation probability.

Theoretically, it has a great advantage in studying the pre-precipitation phase and the phase precipitation process [17]. We chose a computational matrix consisting of $128 \times 128$ lattice points and use dark blue for the $\mathrm{Ni}$ atoms, light green for the $\mathrm{Al}$ atoms and red for the Mo atoms. The lattice site is dark blue if the occupation probability of $\mathrm{Ni}$ is 1.0 , light green if the occupation probability of $\mathrm{Al}$ is 1.0 and red if the occupation probability of Mo is 1.0. The color defines the atoms occupation probability at the lattice site. The figures for the precipitation process are obtained to investigate and analyze the microstructure and how the interatomic potential influences the precipitation process.

\section{Results and Analysis}

\subsection{Microstructure Evolution}

Figure 1 shows the structures of ordered phase and their projections along [010]. Figure 1a is the crystal structure of $\mathrm{L}_{0}(M=1)$ and its projection, while Fig. $1 \mathrm{~b}$ is that for

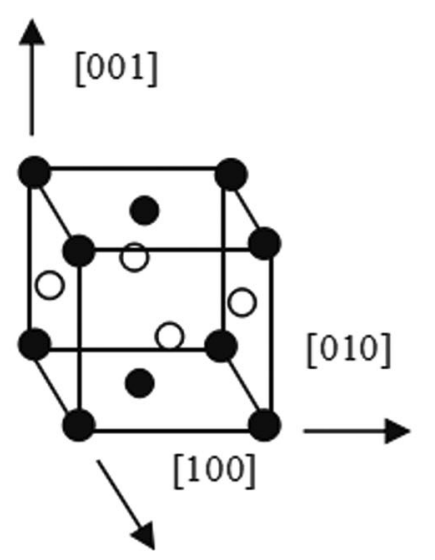

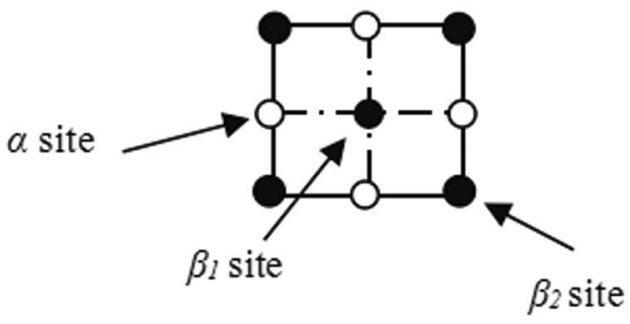

(a)
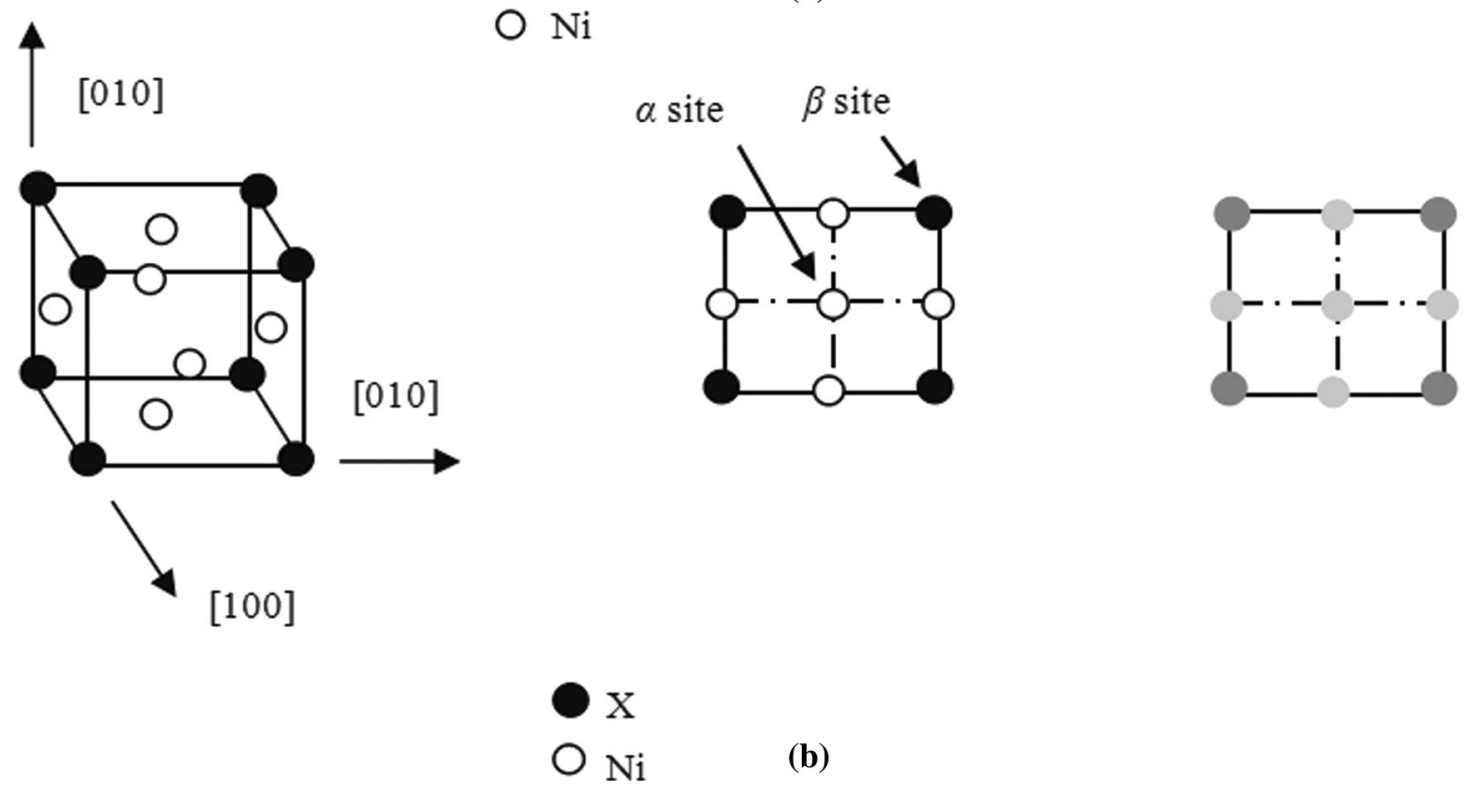

(b)

Fig. 1 Schematic diagrams of the crystal structure and projections along direction [010]: a structure of $\mathrm{L} 1_{0}$ ordered phase; $\mathbf{b}$ structure of $\mathrm{L} 1_{2}\left(\gamma^{\prime}\right.$ phase) ordered phase 
$\mathrm{L1}_{2}$. In Fig. 1, the black dots indicate $\mathrm{X}$ atoms and white dots indicate $\mathrm{Ni}$ atoms.

Figure 2 shows the atomic figures of microstructure evolution of $\mathrm{Ni}_{75} \mathrm{Al}_{14} \mathrm{Mo}_{11}$ alloy under $1073 \mathrm{~K}$. The color assigned to each lattice site is a mixture of red, green, and blue. From Fig. 2a, we can see that there are concentration fluctuations and atom clustering in the disordered matrix at 10,000 steps. It is difficult to distinguish the structures because of low ordered degree of pre-precipitated phase. Ordered structure appears in the matrix since 22,000 steps, which randomly distributes in the matrix with diffuse interfaces among them. We enlarge the ordered phase shown as the box on the lower corner. But at this moment, these ordered particles are unclear and belong to non-stoichiometric ordered phase. In addition, compare Fig. 1a with b, the pre-precipitated phases are respective $\mathrm{L1}_{0}$ and $\mathrm{L1}_{2}$ with lower ordered degree. As time goes on, $\mathrm{L} 1_{0}$ ordered transition phase gradually reduces and $\mathrm{L}_{2}$ phase gradually increases. The $\mathrm{L} 1_{0}$ structure transforms into $\mathrm{L}_{2}$ phase in situ as the occupation portability changes (Fig. 2c, d). At the same time, non-stoichiometric $\mathrm{Ll}_{2}$ phase gradually transforms into stoichiometric $\mathrm{L}_{2}$ phase. Over time, collision and fusion of these ordered phases occur, and some small particles are blended in larger particles nearby which is called coarsening process-small domains disappear, large domains grow up and the interfaces form between the ordered phases. As the time goes continuously, the $\mathrm{L} 1_{0}$ structure turns into $\mathrm{L} 1_{2}$ structure completely, and only $\gamma^{\prime}$ $\left(\mathrm{L1}_{2}\right)$ - ordered phase exists eventually. Therefore, antiphase domain boundary forms among the ordered phases, and the interfaces are clear and narrower. With increasing $\mathrm{Ni}$ occupation probability, $\mathrm{Ni}$ atoms cluster in the antiphase domain boundaries, forming the blue area. At the same time, as the occupation probability of Mo atoms increases gradually, the color of red lattice point gradually deepens as shown in Fig. 2e and f. At $t=500,000$ step, the ordered phases become more uniform and the interface widen.

From the atomic microstructure evolution of $\mathrm{Ni}_{75} \mathrm{Al}_{14}$ $\mathrm{Mo}_{11}$ alloy at $1073 \mathrm{~K}$, we can see two kinds of ordered phases during the aging process and $\mathrm{L}_{0}$ phase transforms into $\mathrm{L} 1_{2}$ phase gradually.

\subsection{Analysis of Occupation Probability}

Through analysis of the occupation probability of atom at different lattice points, we can qualitatively observe the occupation process of the atoms in the precipitation phase and further clarify the structure transformation. The partial enlarged figures of $\mathrm{L} 1_{0}$ phase transforming into $\mathrm{L}_{2}$ phase are shown in Fig. 3.
At 22,000 step, there are two kinds of ordered structures including $\mathrm{L}_{0}$ and $\mathrm{L}_{2}$ as shown in Fig. $3 \mathrm{a}$, b. As time prolonged, the $\mathrm{L1}_{0}$ transforms into $\mathrm{L1}_{2}$ (Fig. 3c) at $t=70,000$. We can find clearly the color of different lattices changes gradually as time going on (Fig. 3b, c, d). In order to further clarify the structural transformation of $\gamma^{\prime}$ phase, we need to compute the occupation probability of $\mathrm{Ni}, \mathrm{Al}$ and Mo atoms at $\alpha, \beta_{1}$ and $\beta_{2}$ lattice points.

Figure 4 shows the variation of occupation probability of $\mathrm{Ni}, \mathrm{Al}$ and $\mathrm{Mo}$ atoms with time at different sites in $\mathrm{Ni}_{75} \mathrm{Al}_{14} \mathrm{Mo}_{11}$ alloy aged at $1073 \mathrm{~K}$. Figure 4 shows that before about 10,000 steps, since the alloy system is in disorder, three occupation probability curves are linear. The occupation probabilities of $\mathrm{Ni}, \mathrm{Al}$, and $\mathrm{Mo}$ atoms at any lattice are the same, which are the initial values.

After 10,000 step, the occupation probability of $\mathrm{Ni}$ atom at $\alpha$ site goes up to about 0.9836 rapidly and then it remains unchanged. While the occupation probabilities of $\mathrm{Al}$ and Mo atoms reduce rapidly until to the minimum values about 0.0153 and 0.0011 , respectively. Therefore, $\mathrm{Ni}$ atoms gradually occupy $\alpha$ site, and the occupation probabilities of $\mathrm{Al}$ and Mo atoms at $\alpha$ site are very small. So $\alpha$ site is occupied by $\mathrm{Ni}$ atom, which is $\mathrm{Al}$ and $\mathrm{Mo}$ atoms' anti-site. Figure $4 \mathrm{~b}$ shows that after 8000 step, the occupation probability of $\mathrm{Ni}$ atom at $\beta_{1}$ rises again to a maximum 0.9829 and remains unchanged after reducing to a certain value, and those of $\mathrm{Al}$ and Mo atoms reduce to small values 0.0154 and 0.0027 , respectively, after going up to a certain value. So $\beta_{1}$ site is occupied by $\mathrm{Ni}$ atom, which is $\mathrm{Al}$ and Mo atoms' anti-site. Comparing Fig. $4 \mathrm{a}$ with $\mathrm{b}$, the occupation probabilities at $\alpha$ and $\beta_{1}$ sites during the atomic initial disordered state and the eventual equilibrium state are the same, but the intermediate process is different. At about $t=22,000$ step shown in Fig. $4 \mathrm{~b}$, both $\mathrm{Al}$ and Mo atom occupation probabilities at $\beta_{1}$ rise to over 0.2 , and at $\beta_{2}$ site rise to over 0.4 . So $\mathrm{L} 1_{0}$ pre-precipitated phase is formed at this stage, which corresponds to Fig. 3a, b. In the same way, as shown in Fig. 4c, we can see that after 8000 step, the occupation probability of $\mathrm{Ni}$ atom gradually reduces to a small equilibrium value and remains the same; the occupation probability of $\mathrm{Al}$ atom slowly rises to a equilibrium value of 0.8422 and that of Mo atoms reduces to the value of 0.1139 , meaning that the $\mathrm{L} 1_{0}$ pre-precipitated phase begins to transform toward $\mathrm{L}_{2}$ phase. Finally, the occupation probabilities at different sites reach the equilibrium values, meaning that the $\mathrm{L} 1_{0}$ pre-precipitated phase is completely transformed into $\mathrm{L}_{2}$ phase. So $\beta_{2}$ sites are occupied by $\mathrm{Al}$ and $\mathrm{Mo}$ atoms and the occupation probability of $\mathrm{Al}$ atom is always bigger than Mo atom. Ni atoms occupy the surface center positions ( $\alpha$ site) and $\mathrm{Al}$, Mo atoms occupy the vertex positions ( $\beta$ site). 

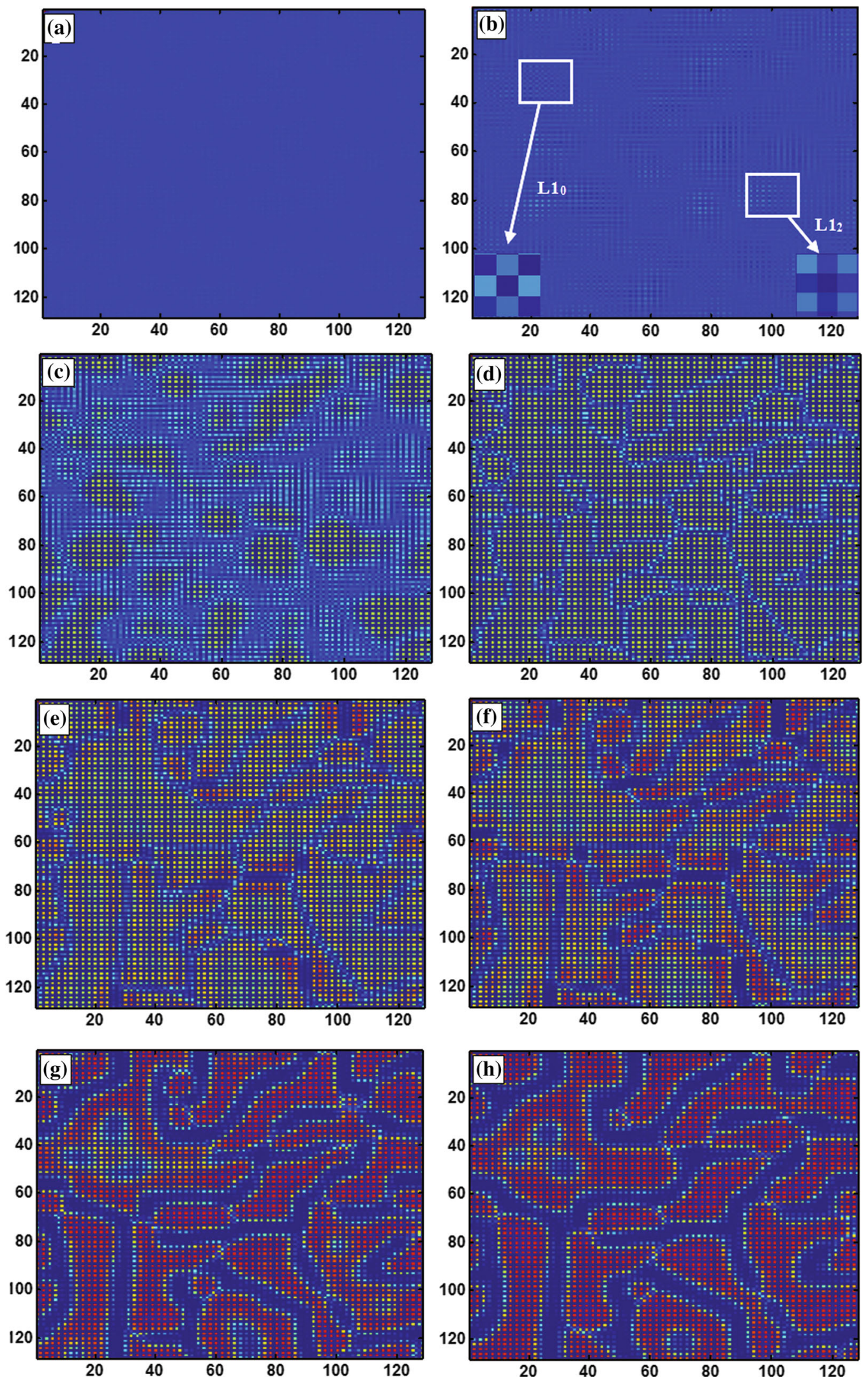

Fig. 2 Atomic figures of microstructure evolution of $\mathrm{Ni}_{75} \mathrm{Al}_{14} \mathrm{Mo}_{11}$ alloy at $1073 \mathrm{~K}$ : a $t=10,000$; b $t=22,000 ; \mathbf{c} t=27,000$; d $t=33,000$; e $t=70,000 ; \mathbf{f} t=100,000 ; \mathbf{g} t=250,000 ; \mathbf{h} t=500,000$ 

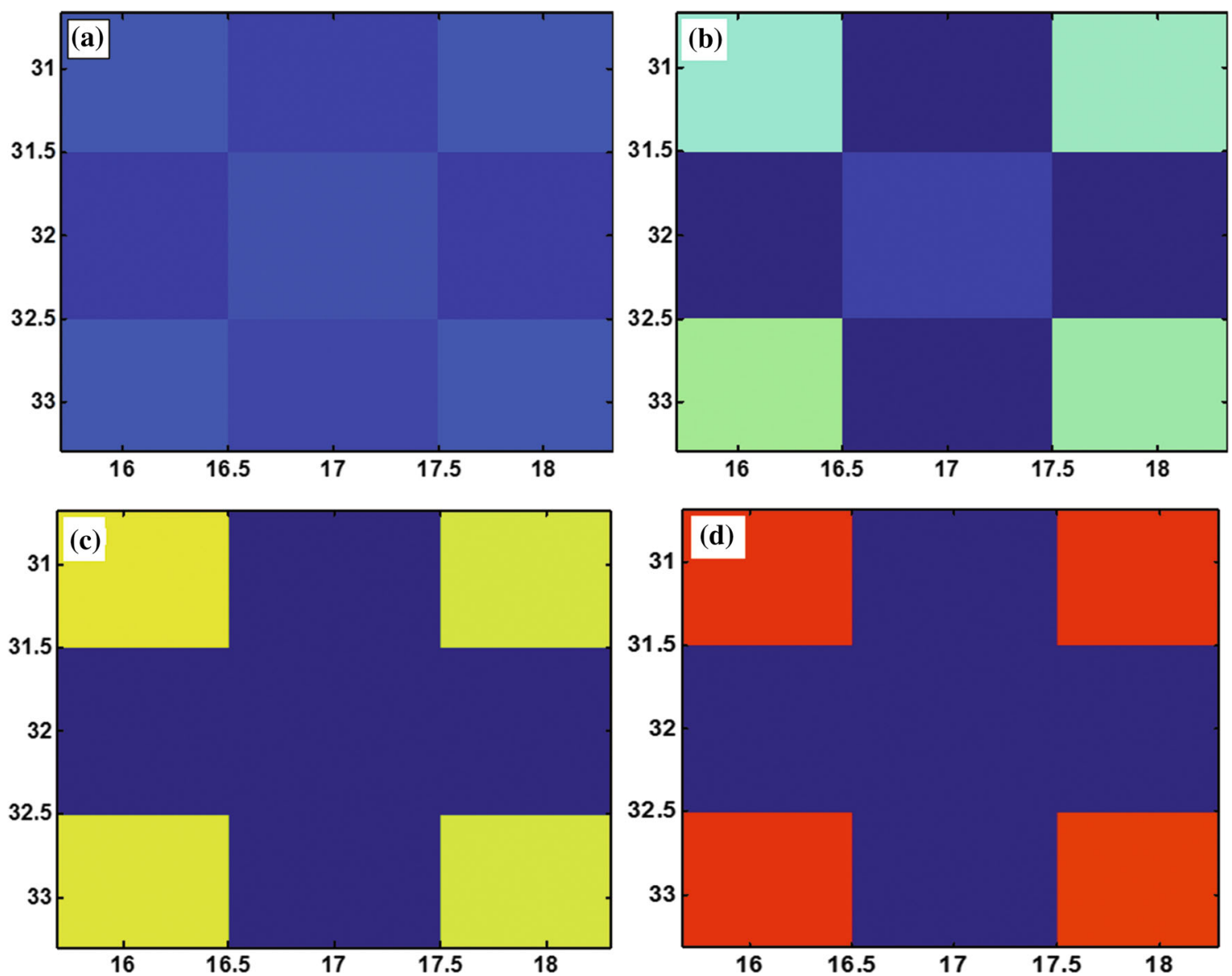

Fig. 3 Partial enlarged figures from $\mathrm{L}_{0}$ phase to $\mathrm{L}_{2}$ phase of $\mathrm{Ni}_{75} \mathrm{Al}_{14} \mathrm{Mo}_{11}$ alloy aged at $1073 \mathrm{~K}$ : a $t=22,000 ; \mathbf{b} t=27,000$; $t=70,000$; d $t=500,000$
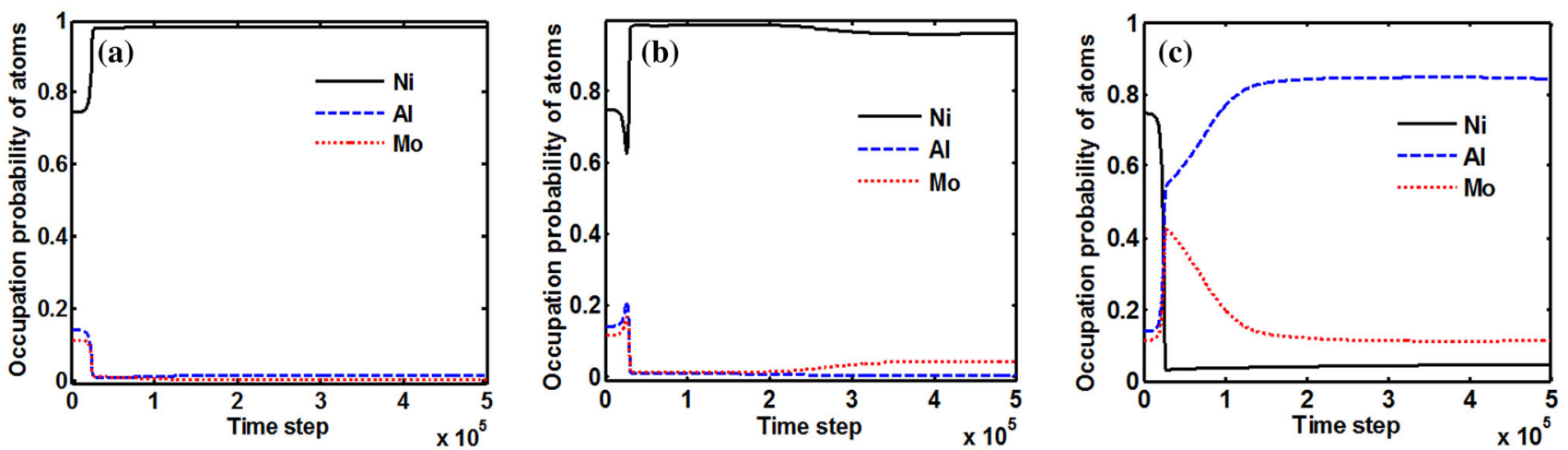

Fig. 4 Variation of the occupation probabilities of $\mathrm{Ni}, \mathrm{Al}$ and $\mathrm{Mo}$ atoms with time at different sites in $\mathrm{Ni}_{75} \mathrm{Al}_{14} \mathrm{Mo}_{11}$ alloy aged at $1073 \mathrm{~K}$ : $\mathbf{a} \alpha$ site; $\mathbf{b} \beta_{1}$ site; $\mathbf{c} \beta_{2}$ site

\subsection{Influences of the Nearest Interatomic Interaction Energy on the $\gamma^{\prime}$ Phase Precipitation Process}

Phase precipitation is a process of atomic diffusion. In other words, the change of phase structure is a variation process of the occupation probability at the lattice. In order to investigate the effect of the interatomic potential on the precipitation process of $\gamma^{\prime}$ phase, we changed the nearest interatomic potential $V_{\mathrm{Ni}-\mathrm{Al}}$ $\left(V_{\mathrm{Ni}-\mathrm{Al}}=122.30 \mathrm{MeV}\right)[18]$ and then computed the occupation probability of $\mathrm{L}_{2}$ phase (Figs. 5, 6), order parameters (Fig. 7) and the volume fraction of $\gamma^{\prime}$ phase (Fig. 8). 

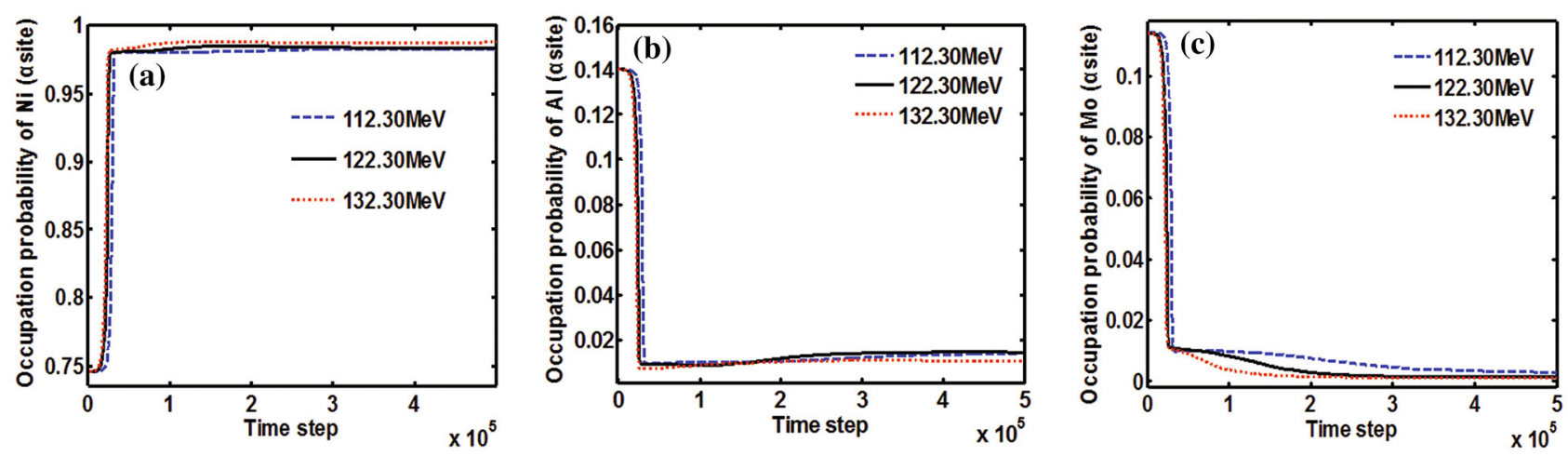

Fig. 5 Variation of the occupation probabilities of different atoms at $\alpha$ site of $\gamma^{\prime}$ phase in $\mathrm{Ni}_{75} \mathrm{Al}_{14} \mathrm{Mo}_{11}$ alloy aged at $1073 \mathrm{~K}$ : a Ni; b $\mathrm{Al} ; \mathbf{c} \mathrm{Mo}$
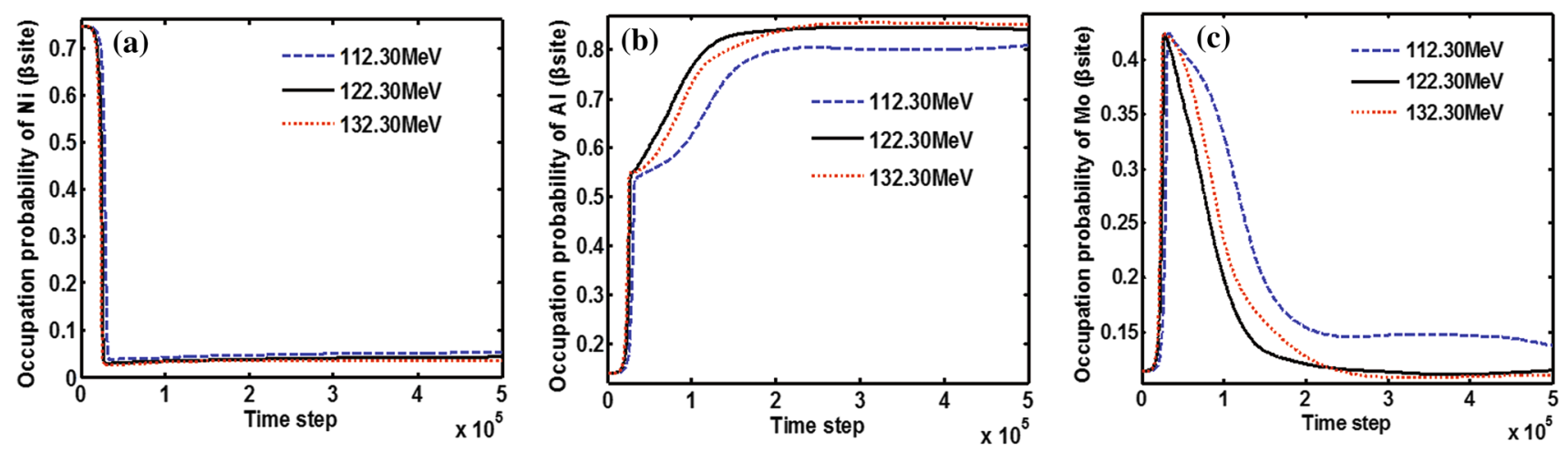

Fig. 6 Variation of the occupation probability of different atoms at $\beta$ site of $\gamma^{\prime}$ phase in $\mathrm{Ni}_{75} \mathrm{Al}_{14} \mathrm{Mo}_{11}$ alloy aged at $1073 \mathrm{~K}$ at different time: a $\mathrm{Ni} ; \mathbf{b ~ A l} ; \mathbf{c} \mathrm{Mo}$

Figure 5 shows the variation of the occupation probabilities of different atoms at $\alpha$ site of $\mathrm{L}_{2}$ phase under the condition that $V_{\mathrm{Ni}-\mathrm{Al}}$ is changed $\pm 10 \mathrm{MeV}$. From Fig. 5, we can find that when the $V_{\mathrm{Ni}-\mathrm{Al}}$ increases $10 \mathrm{MeV}$, the occupation probability of $\mathrm{Ni}$ atom at $\alpha$ site increases gradually after about 7000 steps and then goes up rapidly to about 0.9880 and finally remains unchanged, which is bigger than value acquired when the $V_{\mathrm{Ni}-\mathrm{Al}}$ is unchanged (Fig. 5a). While the occupation probabilities of $\mathrm{Al}$ and Mo atoms decrease after about 7000 steps and then go down rapidly to about 0.0112 and about 0.008 , respectively (Fig. 5b, c). It is not hard to find that the curves shift to the left, and the time point that the occupation probability begins to go up earlier.

The changing process of the occupation probability of the atoms at $\beta$ site of $\mathrm{L}_{2}$ phase is given in Fig. 6 when $V_{\mathrm{Ni}-\mathrm{Al}}$ is changed $\pm 10 \mathrm{MeV}$. In the same way, from Fig. 6 we can find that when the $V_{\mathrm{Ni}-\mathrm{Al}}$ increases $10 \mathrm{MeV}$, the occupation probability of $\mathrm{Ni}$ atom at $\beta$ site decreases gradually after about 7000 steps and then goes up rapidly to about 0.0370 and finally remains unchanged for a long time, which is smaller than the value acquired when the $V_{\mathrm{Ni}-\mathrm{Al}}$ is unchanged (Fig. 6a). While the occupation probability of $\mathrm{Al}$ atom increases gradually after about 7000 steps and then goes up rapidly to a certain value and then keeps going up slowly to about 0.8524 at last (Fig. 5b, c), which is bigger than the value acquired when the $V_{\mathrm{Ni}-\mathrm{Al}}$ is unchanged (Fig. 6b). The occupation probability of Mo atom increases gradually after about 7000 steps and then goes up rapidly to a certain value at first and then goes down slowly to about 0.1116 eventually. With $V_{\mathrm{Ni}-\mathrm{Al}}$ increased $10 \mathrm{MeV}$, the occupation probability of Mo atom becomes smaller. From the occupation probability curves in Fig. 5, it can be seen that all the curves shift to the left, too.

From the above observation and analysis, it can be concluded that during the $\gamma^{\prime}$ phase precipitation process in $\mathrm{Ni}_{75} \mathrm{Al}_{14} \mathrm{Mo}_{11}$ alloy aged at $1073 \mathrm{~K}$, the increase in nearest interatomic interaction energy $V_{\mathrm{Ni}-\mathrm{Al}}$ can promote the occupation probability of $\mathrm{Ni}$ atom at $\alpha$ site and the occupation probability of $\mathrm{Al}$ atom at $\beta$ site, meaning the precipitated phase is not changed. Moreover, the increase in $V_{\mathrm{Ni}-\mathrm{Al}}$ makes the transformation time point go ahead meaning the incubation period of $\gamma^{\prime}$ phase becomes shorter. So the increase in nearest interatomic interaction energy $V_{\mathrm{Ni}-\mathrm{Al}}$ has a promoting effect on the formation of $\gamma^{\prime}$ phase.

The changes of $\mathrm{Al}$ and Mo average long-range-order parameter (ALROP) and average composition order 

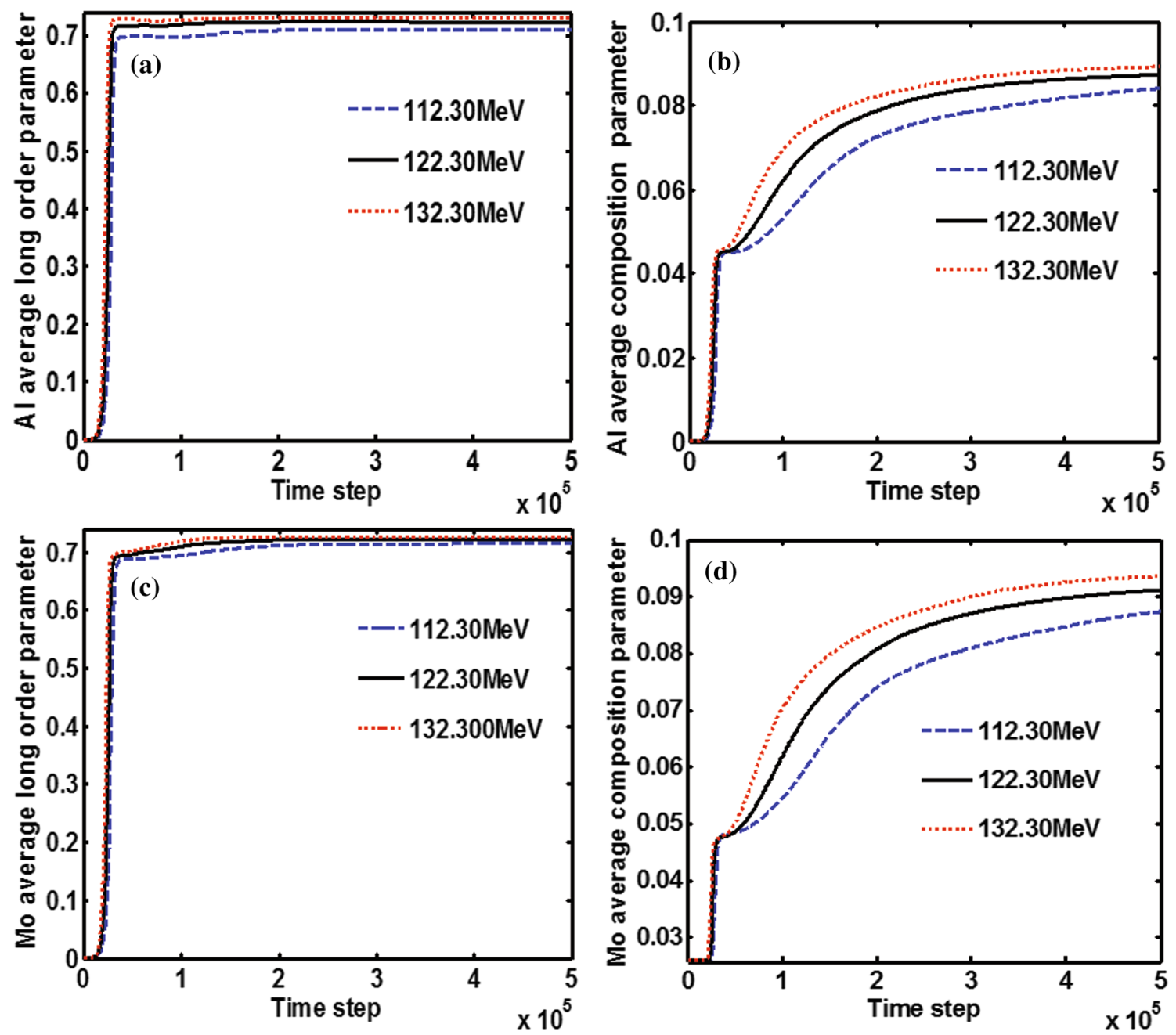

Fig. 7 Changes of the average long-range-order parameter $(\mathbf{a}, \mathbf{c})$ and average composition parameter (b, d) of $\mathrm{Al}$ and $\mathrm{Mo}$ with time

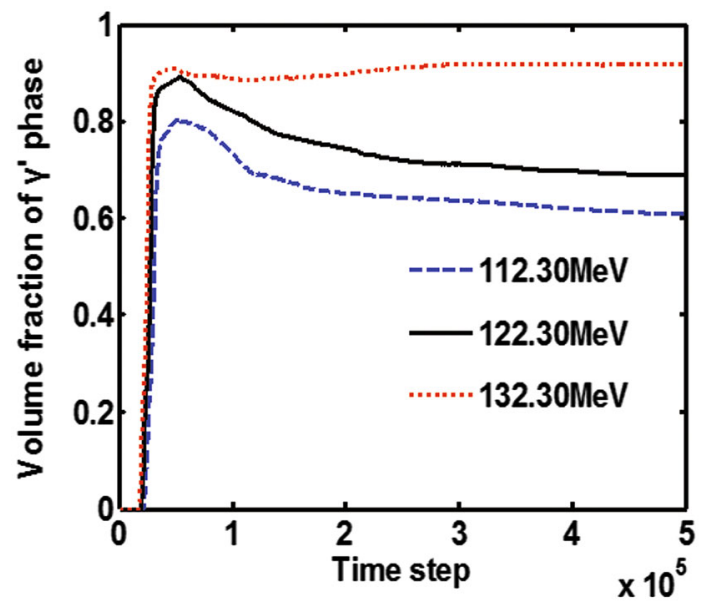

Fig. 8 Effect of the nearest interatomic interaction energy on the volume fractions of $\gamma^{\prime}$ phase

parameter (ACOP) with time in $\mathrm{Ni}_{75} \mathrm{Al}_{14} \mathrm{Mo}_{11}$ alloy aged at $1073 \mathrm{~K}$ are shown in Fig. 7. It shows that clustering process and the ordering process of the atom exist in the phase precipitation process. The average long rang order parameter and average composition order parameter present the atomic ordering-disordering transformation process and clustering process of the atom, respectively. So we can use the ordering of $\mathrm{Al}$ and Mo atoms to present the ordering of $\gamma^{\prime}$ phase. From Fig. 7a and b, we know the formation process of $\gamma^{\prime}$ phase has incubation period. In addition, with $V_{\mathrm{Ni}-\mathrm{Al}}$ increased by $10 \mathrm{MeV}$, both the average long-range-order parameter and average composition order parameter of $\mathrm{Al}$ atom increase with time and the curves shift to the left. Figure $7 \mathrm{c}$ and $\mathrm{d}$ shows the variation of average long rang order parameter and average composition parameter of $\mathrm{Mo}$ with time in $\mathrm{Ni}_{75} \mathrm{Al}_{14} \mathrm{Mo}_{11}$ alloy aged at $1073 \mathrm{~K}$. It comes to the same conclusion in the same way.

The average long order parameter can reflect the order degree of the precipitated phase and the average composition parameter can reflect the clustering. By these two parameters, the phase precipitation process including the incubation period can be observed clearly. From Fig. 7 and 
above analysis, it can be seen clearly that the increase in the nearest interatomic potential $V_{\mathrm{Ni}-\mathrm{Al}}$ is helpful for the clustering and the ordering of $\mathrm{Al}$ and Mo. The incubation period is shortened. So it has a promoting effect on the formation of $\gamma^{\prime}$ phase.

In addition, we also studied how the $V_{\mathrm{Ni}-\mathrm{Al}}$ affects the volume fractions of $\gamma^{\prime}$ phase. Figure 8 shows the effect of the nearest interatomic potential on the volume fraction of $\gamma^{\prime}$ phase. When $V_{\mathrm{Ni}-\mathrm{Al}}$ is increased by $10 \mathrm{MeV}$, the volume fraction curve shifts to the left and the volume fraction of $\gamma^{\prime}$ phase goes up to about $91.81 \%$, which increases $33.31 \%$ compared to the original volume fraction (68.87\%). Therefore, we come to the conclusion that the increase in the nearest interatomic potential $V_{\mathrm{Ni}-\mathrm{Al}}$ can promote the formation of $\gamma^{\prime}$ phase.

\section{Discussion}

Chen et al. [19] thought that the main factor that influences the second phase precipitation is the driving force leading to the change of local long-range-ordering fluctuations. $\delta \eta(r)$, the second free energy parameter introduced, is related to the corresponding long-range-order parameters:

$\Delta f \rightarrow \int\left(\frac{\partial^{2} f}{\partial \eta^{2}}\right)_{\eta=0}(\delta \eta)^{2} d^{3} r \rightarrow C\left(T-T_{-}\right) \int(\delta \eta)^{2} d^{3} r$,

where $C$ is a constant and $T$ is the ordering instability temperature below which $\left(\partial^{2} f / \partial \eta^{2}\right)_{\eta=0}$ can be negative. Therefore, it is the driving force for local long-range-order fluctuation which is relevant to the rate of ordering. Equation (6) also shows that the driving force for local fluctuation is proportional to the undercooling below the ordering instability line, $\left(T-T_{-}\right)$.

Khachaturyan [20] pointed out the relationship of $T_{-}$ and $V_{\alpha \beta}\left(k_{0}\right)$.

$T_{-}=\frac{\varphi(c)}{k_{\mathrm{B}}} \cdot v_{\alpha \beta}\left(k_{0}\right)$,

where $\varphi(c)$ is a function, which is associated with atomic concentration $c . V_{\alpha \beta}\left(k_{0}\right)$ is the interatomic potential. $V_{\mathrm{Ni}-\mathrm{Al}}$ is the interatomic potential of $\mathrm{L}_{2}$ phase. From Eq. (7), we can find that the change of $V_{\mathrm{Ni}-\mathrm{Al}}$ can only change the ordering instability temperature $T_{-}$. And it is easy to find that the undercooling $\left(T-T_{-}\right)$of $\mathrm{L}_{2}$ will increase along with increasing $V_{\mathrm{Ni}-\mathrm{Al}}$. And then the driving force increases.

According to this theory above, when the $V_{\mathrm{Ni}-\mathrm{Al}}$ increases, the undercooling $\left(T-T_{-}\right)$of $\mathrm{L}_{2}$ will increase following, and then, the driving force of $\mathrm{L}_{2}$ phase will become stronger. So the increase in $V_{\mathrm{Ni}-\mathrm{Al}}$ can speed up the precipitation of ordered phase. Thus, in the $\gamma^{\prime}$ phase precipitation process of $\mathrm{Ni}_{75} \mathrm{Al}_{14} \mathrm{Mo}_{11}$ alloy aged at $1073 \mathrm{~K}$, it shows that the incubation period of $\gamma^{\prime}$ phase becomes shorter and the volume fraction of $\gamma^{\prime}$ phase goes up greatly. Here, we just combine these two theories to speculate some phenomena presented in this simulation. Further studies are needed to explain these changes.

\section{Conclusions}

Two kinds of ordered phases $\mathrm{L}_{0}$ and $\mathrm{L} 1_{2}$ precipitate from the systems. Both of them are occupied by $\mathrm{Ni}, \mathrm{Al}$ and $\mathrm{Mo}$ atoms. In addition, the $\mathrm{L}_{0}$ transforms into $\mathrm{L}_{2}$ as the occupation portability changing.

In the $\gamma^{\prime}$ phase ( $\mathrm{L} 1_{2}$ phase), $\alpha$ site is occupied by $\mathrm{Ni}$ atom and $\mathrm{Al}$ and anti-site defect Mo, while $\mathrm{Al}$ and Mo atoms occupy $\beta$ sites together with anti-site defect $\mathrm{Ni}$. The occupation probability of $\mathrm{Al}$ atom is always bigger than the occupation probability of Mo atom.

The increase in the nearest interatomic potential $V_{\mathrm{Ni}-\mathrm{Al}}$ is helpful for clustering and ordering of $\mathrm{Al}$ and $\mathrm{Mo}$ atoms, and clustering and ordering of atom appear earlier. The volume fraction of $\gamma^{\prime}$ phase increases as $V_{\mathrm{Ni}-\mathrm{Al}}$ increases, too. So the increase in $V_{\mathrm{Ni}-\mathrm{Al}}$ can shorten the incubation period of $\gamma^{\prime}$ phase and promote phase formation.

Acknowledgments This work was financially supported by the National Natural Science Foundation of China (Nos. 51,204,147 and 51274175) and International Cooperation Project Supported by Ministry of Science and Technology of China (No. 2014DFA50320), International Science and Technology Cooperation Project of Shanxi Province (Nos. 2013081017 and 2012081013).

\section{References}

[1] Y. Ustinovshikov, J. Alloys Compd. 582, 119 (2014)

[2] S.F. Miao, Z. Chen, Y.X. Wang, C. Xu, R. Ma, M.Y. Zhang, Acta Metall. Sin.45, 630 (2009) (in Chinese)

[3] Y.L. Lu, Z. Chen, X. Li, Comput. Mater. Sci. 99, 247 (2015)

[4] Y.L. Lu, D.W. Jia, T.T. Hu, Z. Chen, L.C. Zhang, Superlattices Microstruct. 66, 105 (2014)

[5] J. Zhang, Z. Chen, Y.X. Wang, L.J. Tong, China Sci. Technol. Pap 16, 1894 (2015)

[6] W.P. Dong, Y.X. Wang, K. Yang, Chin. Sci. Bull. 56, 2055 (2011)

[7] W.P. Dong, Y.X. Wang, Z. Chen, K. Yang, Trans. Nonferrous Met. Soc. China 21, 1105 (2011)

[8] Y. Zhao, Z. Chen, Y.X. Wang, M. Zhang, Acta Metall. Sin. 45, 635 (2009) (in Chinese)

[9] R.C. Pasianot, L. Malerba, J. Nucl. Mater. 360, 118 (2007)

[10] P. Oramus, C. Massobrio, M. Kozlowski, Comput. Mater. Sci. 47, 186 (2003)

[11] A.G. Khachaturyan, Theory of Structural Transformation in Solids (Wiley, New York, 1983), p. 129 
[12] L.Q. Chen, A.G. Khachaturyan, Scr. Metall. Mater. 25, 61 (1991)

[13] R. Poduri, L.Q. Chen, Acta Mater. 46, 1719 (1998)

[14] L.Q. Chen, W. Yang, Phys. Rev. B 50, 15752 (1994)

[15] L.Q. Chen, Annu. Rev. Mater. Res. 32, 113 (2002)

[16] Y. Wang, A.G. Khachaturyan, Acta Metall. Mater. 43, 1837 (1995)
[17] J.W. Cahn, J.E. Hillard, Chem. Phys. 28, 258 (1958)

[18] N.T. Wilson, M.S. Bailey, R.L. Johnston, J. Inorg. Chim. Acta 359, 3649 (2006)

[19] R. Poduri, L.Q. Chen, Acta Mater. 46, 1719 (1998)

[20] A.G. Khachaturyan, Theory of Structural Transformations in Solids (Wiley, New York, 1983), p. 133 